\title{
POSITIVE DEPENDENCE OF SIGNALS
}

\author{
MICHEL DENUIT, ${ }^{*}$ Université Catholique de Louvain
}

\begin{abstract}
In this paper we further investigate the problem considered by Mizuno (2006) in the special case of identically distributed signals. Specifically, we first propose an alternative sufficient condition of crossing type for the convex order to hold between the conditional expectations given signal. Then, we prove that the bivariate $(2,1)$-increasing convex order ensures that the conditional expectations are ordered in the convex sense. Finally, the $L^{2}$ distance between the quantity of interest and its conditional expectation given signal (or expected conditional variance) is shown to decrease when the strength of the dependence increases (as measured by the (2,1)-increasing convex order).
\end{abstract}

Keywords: Concordance order; supermodularity; convex order; conditional expectation; bivariate order of increasing-convex type

2010 Mathematics Subject Classification: Primary 60E15

\section{Introduction and motivation}

There are numerous situations in which we cannot observe directly a variable $X$ of interest but we only have at our disposal a signal $S$ for $X$. The signal $S$ is correlated to $X$ so that observing $S$ brings some information about the hidden $X$ : the more $S$ is correlated to $X$, the more information it contains. Hence, we prefer signals $S$ as perfectly correlated to $X$ as possible. Considering two identically distributed signals $S_{1}$ and $S_{2}$ for $X$, the strength of the dependence in the pairs $\left(X, S_{1}\right)$ and $\left(X, S_{2}\right)$ can be compared with appropriate bivariate stochastic order relations, such as the concordance order for instance.

We can also compare signals based on the property that more informative signals lead to greater variability of the conditional expectation $\mathrm{E}[X \mid S]$. If the signal $S$ is independent of $X$ then the variance of $\mathrm{E}[X \mid S]=\mathrm{E}[X]$ is 0 so that $S$ does not contain any information about $X$. On the other hand, if the signal is perfect, that is, if $S=X$, then the variance of $E[X \mid S]$ is maximum, being equal to the variance of $X$. The convex order is often used in applied probability to compare the variability inherent to probability distributions beyond standard deviations. Recall that a random variable $Y$ is said to be smaller than another random variable $Z$ in the convex order, henceforth denoted as $Y \preceq_{\mathrm{cx}} Z$, if $\mathrm{E}[Y]=\mathrm{E}[Z]$ and $\mathrm{E}\left[(Y-t)_{+}\right] \leq$ $\mathrm{E}\left[(Z-t)_{+}\right]$for all $t \in \mathbb{R}$. The name convex order comes from the fact that $Y \preceq_{\mathrm{cx}} Z$ if and only if $\mathrm{E}[g(Y)] \leq \mathrm{E}[g(Z)]$ for all the convex functions $g$ for which the expectations exist. For more details, we refer the reader to, e.g. Shaked and Shanthikumar (2007) or to Denuit et al. (2005). Here, we consider that a signal $S_{2}$ is more informative than another signal $S_{1}$ if $\mathrm{E}\left[X \mid S_{1}\right] \preceq_{\mathrm{cx}} \mathrm{E}\left[X \mid S_{2}\right]$. The literature about auction theory says that $S_{2}$ is more integral precise than $S_{1}$ in such a case. See Ganuza and Penalva (2010).

In this paper we show that these two approaches for comparing signals $S_{1}$ and $S_{2}$ are essentially equivalent: if the pair $\left(X, S_{2}\right)$ is more positively dependent than the pair $\left(X, S_{1}\right)$ then

Received 5 February 2010; revision received 22 May 2010.

* Postal address: Institut de Statistique, Biostatistique \& Sciences Actuarielles - ISBA, Université Catholique de Louvain, B-1348 Louvain-la-Neuve, Belgium. Email address: michel.denuit@uclouvain.be 
there is more information in $S_{2}$ about $X$ compared to $S_{1}$. We restrict our analysis to identically distributed signals so that we concentrate on the dependence structure of $\left(X, S_{1}\right)$ and $\left(X, S_{2}\right)$, avoiding any marginal effect.

Following Mizuno (2006), let us consider the trivariate nonnegative random vector $\left(X, S_{1}\right.$, $S_{2}$ ), where $S_{1}$ and $S_{2}$ are interpreted as noisy signals of the unobservable random variable $X$. Let $F$ denote the distribution function of $X$, i.e. $F(x)=\operatorname{Pr}[X \leq x]$, and let $G$ denote the common distribution function of $S_{1}$ and $S_{2}$, i.e. $G(s)=\operatorname{Pr}\left[S_{1} \leq s\right]=\operatorname{Pr}\left[S_{2} \leq s\right]$. Furthermore, $G_{i}(\cdot \mid x)$ is the conditional distribution function of $S_{i}$ given $X=x, i=1,2$, and $m_{i}(s)=\mathrm{E}\left[X \mid S_{i}=s\right]$ is the conditional expectation of $X$ given $S_{i}=s, i=1,2$. Throughout the paper, we assume, as in Mizuno (2006), that both $m_{1}$ and $m_{2}$ are nondecreasing. Mizuno (2006) proved that if the function $x \mapsto G_{1}\left(s_{1} \mid x\right)-G_{2}\left(s_{2} \mid x\right)$ changes sign at most once from negative to positive as $x$ increases, then $m_{1}\left(S_{1}\right)$ precedes $m_{2}\left(S_{2}\right)$ in the convex order.

In this paper we first propose an alternative sufficient condition of crossing type for $m_{1}\left(S_{1}\right)$ and $m_{2}\left(S_{2}\right)$ to be ordered in the convex sense. As suggested in Mizuno (2006, p. 1185), the assumptions of his Proposition 1 are strong and can be relaxed when the analysis is restricted to identically distributed signals. In fact, we show that if the bivariate $(2,1)$-increasing convex order introduced in Denuit et al. (1999) holds between $\left(X, S_{1}\right)$ and $\left(X, S_{2}\right)$, then $m_{1}\left(S_{1}\right)$ and $m_{2}\left(S_{2}\right)$ are ordered in the convex sense. This result turns out to be useful for the applications as most bivariate models can be ordered in the bivariate (2,1)-increasing convex order (which is weaker than the concordance order, or $(1,1)$-increasing convex order). Finally, we examine the closeness (in the $L^{2}$ distance) of $X$ to its conditional expectation given signal when the strength of dependence is increased (in the sense of the $(2,1)$-increasing convex order).

\section{Crossing-type condition for the conditional expectations}

Let us now propose an alternative to the sufficient condition in Proposition 1 of Mizuno (2006) in the special case of identically distributed signals $S_{1}$ and $S_{2}$. Instead of considering the function $x \mapsto G_{1}\left(s_{1} \mid x\right)-G_{2}\left(s_{2} \mid x\right)$ for arbitrary $s_{1}$ and $s_{2}$, we use here the difference $s \mapsto m_{2}(s)-m_{1}(s)$ of the conditional expectations of $X$ given signals. In the next result, we show that it suffices that the difference $m_{1}-m_{2}$ exhibits a single sign change for the conditional expectations $m_{1}\left(S_{1}\right)$ and $m_{2}\left(S_{2}\right)$ to be ordered in the convex sense.

Proposition 2.1. Assume that $m_{1}$ and $m_{2}$ are nondecreasing. If $s \mapsto m_{2}(s)-m_{1}(s)$ changes sign at most once from negative to positive as $s$ increases, that is,

$$
m_{2}(s) \geq m_{1}(s) \quad \Longrightarrow \quad m_{2}\left(s^{\prime}\right) \geq m_{1}\left(s^{\prime}\right) \quad \text { for all } s \leq s^{\prime},
$$

then $m_{1}\left(S_{1}\right) \preceq \mathrm{cx} m_{2}\left(S_{2}\right)$.

Proof. Note that $\mathrm{E}\left[m_{1}\left(S_{1}\right)\right]=\mathrm{E}\left[m_{2}\left(S_{2}\right)\right]=\mathrm{E}[X]$. Condition (2.1) ensures that the functions $m_{1}$ and $m_{2}$ cross at most once, and that $m_{2}$ dominates $m_{1}$ for sufficiently large arguments. Hence, the distribution functions of $m_{1}\left(S_{1}\right)$ and $m_{2}\left(S_{2}\right)$ cross exactly once (because of equal expectations), the distribution function of $m_{1}\left(S_{1}\right)$ dominating for sufficiently large arguments. By Theorem 3.A.44 of Shaked and Shanthikumar (2007), this ensures that $m_{1}\left(S_{1}\right) \preceq \mathrm{cx} m_{2}\left(S_{2}\right)$, as announced.

The single crossing condition (2.1) is not on the conditional distributions, as in Mizuno (2006), but involves the conditional expectations. It is useful in some applications, for instance in auction theory. A related result has been obtained in Ganuza and Penalva (2010, Lemma 1) 
where the dispersive order between $m_{1}\left(S_{1}\right)$ and $m_{2}\left(S_{2}\right)$ is obtained under a stronger condition than (2.1).

\section{Bivariate (2,1)-increasing convex order}

For some positive integer $s$, let $U_{s-\text { icx }}$ be the class of measurable functions with nonnegative derivatives of degrees 1 to $s$. Recall from Denuit et al. (1998) that given two random variables $Y$ and $Z, Y$ is said to be smaller than $Z$ in the $s$-increasing convex sense, denoted by $Y \preceq{ }_{s} Z$, when $\mathrm{E}[g(Y)] \leq \mathrm{E}[g(Z)]$ for all the functions $g \in U_{s-\text { icx }}$ such that the expectations exist. For $s=1$, ' $\preceq_{1}$ ' coincides with the usual stochastic dominance. For $s=2, \preceq_{2}$ ' is the increasing convex order. Furthermore, $Y \preceq_{2} Z$ and $\mathrm{E}[Y]=\mathrm{E}[Z]$ if and only if $Y \preceq_{\mathrm{cx}} Z$.

Let us now consider the bivariate case and denote by $g^{(i, j)}$ the $(i, j)$ th mixed partial derivative of $g$ with respect to $x_{1}$ and $x_{2}$, that is, $g^{(i, j)}=\left(\partial^{i+j} / \partial x_{1}^{i} \partial x_{2}^{j}\right) g$. For some positive integers $s_{1}$ and $s_{2}$, let $U_{\left(s_{1}, s_{2}\right)-\text { icx }}$ be the class of measurable functions $g$ such that $g^{\left(k_{1}, k_{2}\right)} \geq 0$ for all $k_{1}=0, \ldots, s_{1}$ and $k_{2}=0, \ldots, s_{2}$, such that $k_{1}+k_{2} \geq 1$. Recall from Denuit et al. (1999) that $\left(X, S_{1}\right)$ is said to be smaller than $\left(X, S_{2}\right)$ in the bivariate $\left(s_{1}, s_{2}\right)$-increasing convex order, denoted by $\left(X, S_{1}\right) \preceq_{\left(s_{1}, s_{2}\right)}\left(X, S_{2}\right)$, when $\mathrm{E}\left[g\left(X, S_{1}\right)\right] \leq \mathrm{E}\left[g\left(X, S_{2}\right)\right]$ for all $g \in U_{\left(s_{1}, s_{2}\right)-\text { icx }}$ such that the expectations exist.

For $s_{1}=s_{2}=1$, ' $\preceq_{(1,1)}$ ' coincides with the concordance order. Concordance conveys the idea of clustering of large and small events. Large and small values tend to be more often associated under the distribution that dominates the other one in the concordance order. The ' $\preceq_{(1,1)}$ ' order can be characterized by

$$
\begin{aligned}
\left(X, S_{1}\right) \preceq(1,1) & \left(X, S_{2}\right) \\
& \Longleftrightarrow \operatorname{Pr}\left[X>t_{1}, S_{1}>t_{2}\right] \leq \operatorname{Pr}\left[X>t_{1}, S_{2}>t_{2}\right] \quad \text { for all } t_{1}, t_{2} \\
& \Longleftrightarrow \mathrm{E}\left[h_{1}(X) h_{2}\left(S_{1}\right)\right] \leq \mathrm{E}\left[h_{1}(X) h_{2}\left(S_{2}\right)\right] \quad \text { for all nondecreasing } h_{1}, h_{2} \geq 0 \\
& \Longleftrightarrow \mathrm{E}\left[g\left(X, S_{1}\right)\right] \leq \mathrm{E}\left[g\left(X, S_{2}\right)\right] \quad \text { for all nondecreasing } g \text { such that } g^{(1,1)} \geq 0 .
\end{aligned}
$$

Since $\left(X, S_{1}\right)$ and $\left(X, S_{2}\right)$ have identical marginal distributions, we also have

$$
\begin{aligned}
\left(X, S_{1}\right) & \preceq(1,1)\left(X, S_{2}\right) \\
& \Longleftrightarrow \operatorname{cov}\left[h_{1}(X), h_{2}\left(S_{1}\right)\right] \leq \operatorname{cov}\left[h_{1}(X), h_{2}\left(S_{2}\right)\right] \quad \text { for all nondecreasing } h_{1}, h_{2} \geq 0 \\
& \Longleftrightarrow \operatorname{Pr}\left[X>t_{1} \mid S_{1}>t_{2}\right] \leq \operatorname{Pr}\left[X>t_{1} \mid S_{2}>t_{2}\right] \\
& \quad \text { for all } t_{1} \text { and } t_{2} \text { provided that } \operatorname{Pr}\left[S_{i}>t_{2}\right]>0, i=1,2 .
\end{aligned}
$$

The latter inequality intuitively means that the knowledge that $S_{2}$ is large (that is, $S_{2}>t_{2}$ ) increases the probability that $X$ is also large (that is, $\left.X>t_{1}\right)$ compared to $\left(X, S_{1}\right)$.

Now, for $s_{1}=2$ and $s_{2}=1$, the stochastic order relation ' $\preceq_{(2,1)}$ ' is weaker than the concordance order ' $\preceq_{(1,1)}$ '. Denoting as $\mathbf{1}(A)$ the indicator function of the event $A$ (equal to 1 if $A$ is realized and to 0 otherwise), and remembering that $\left(X, S_{1}\right)$ and $\left(X, S_{2}\right)$ have identical marginal distributions, it can be characterized by

$$
\begin{aligned}
\left(X, S_{1}\right) & \preceq(2,1)\left(X, S_{2}\right) \\
& \Longleftrightarrow \int_{t_{1}}^{\infty} \operatorname{Pr}\left[X>\xi, S_{1}>t_{2}\right] \mathrm{d} \xi \leq \int_{t_{1}}^{\infty} \operatorname{Pr}\left[X>\xi, S_{2}>t_{2}\right] \mathrm{d} \xi \quad \text { for all } t_{1}, t_{2} \\
& \Longleftrightarrow \mathrm{E}\left[\left(X-t_{1}\right)_{+} \mathbf{1}\left(S_{1}>t_{2}\right)\right] \leq \mathrm{E}\left[\left(X-t_{1}\right)_{+} \mathbf{1}\left(S_{2}>t_{2}\right)\right] \quad \text { for all } t_{1}, t_{2} \\
& \Longleftrightarrow \mathrm{E}\left[h_{1}(X) h_{2}\left(S_{1}\right)\right] \leq \mathrm{E}\left[h_{1}(X) h_{2}\left(S_{2}\right)\right]
\end{aligned}
$$

for all nondecreasing $h_{1}, h_{2} \geq 0$ with $h_{1}$ convex 


$$
\begin{aligned}
& \Longleftrightarrow \quad \operatorname{cov}\left[h_{1}(X), h_{2}\left(S_{1}\right)\right] \leq \operatorname{cov}\left[h_{1}(X), h_{2}\left(S_{2}\right)\right] \\
& \quad \text { for all nondecreasing } h_{1}, h_{2} \geq 0 \text { with } h_{1} \text { convex } \\
& \Longleftrightarrow \quad \mathrm{E}\left[g\left(X, S_{1}\right)\right] \leq \mathrm{E}\left[g\left(X, S_{2}\right)\right] \\
& \quad \text { for all nondecreasing } g \text { such that } g^{(2,0)} \geq 0, g^{(1,1)} \geq 0, \text { and } g^{(2,1)} \geq 0 \\
& \\
& \begin{array}{r}
\mathrm{E}\left[\left(X-t_{1}\right)_{+} \mid S_{1}>t_{2}\right] \leq \mathrm{E}\left[\left(X-t_{1}\right)_{+} \mid S_{2}>t_{2}\right] \\
\text { for all } t_{1} \text { and } t_{2} \text { provided that } \operatorname{Pr}\left[S_{i}>t_{2}\right]>0, i=1,2 .
\end{array}
\end{aligned}
$$

Inequality (3.1) shows that $\left(X, S_{1}\right) \preceq_{(2,1)}\left(X, S_{2}\right)$ means that the knowledge that $S_{2}$ is large (that is, $\left.S_{2}>t_{2}\right)$ increases the average part of $X$ above any threshold $t_{1}$ compared to $\left(X, S_{1}\right)$. This also means that the conditional distribution of $X$ given $S_{2}>t_{2}$ dominates the conditional distribution of $X$ given $S_{1}>t_{2}$ in the ' $\preceq 2$ ' order.

We are now in a position to establish the following result.

Proposition 3.1. If $\left(X, S_{1}\right) \preceq_{(2,1)}\left(X, S_{2}\right)$ then $m_{1}\left(S_{1}\right) \preceq_{\mathrm{cx}} m_{2}\left(S_{2}\right)$.

Proof. From Shaked and Shanthikumar (2007, Equation (3.A.41)) we know that $Y \preceq_{\mathrm{cx}} Z$ if and only if $\mathrm{E}[Y]=\mathrm{E}[Z]$ and

$$
\mathrm{E}\left[Y \mid Y \geq F_{Y}^{-1}(p)\right] \leq \mathrm{E}\left[Z \mid Z \geq F_{Z}^{-1}(p)\right] \text { for all } p \in[0,1),
$$

where $F_{Y}^{-1}$ and $F_{Z}^{-1}$ are the quantile functions associated with the distribution functions $F_{Y}$ and $F_{Z}$, respectively. Denoting by $G_{m_{1}\left(S_{1}\right)}$ the distribution function of $m_{1}\left(S_{1}\right)$, we have

$$
\begin{aligned}
\mathrm{E}\left[m_{1}\left(S_{1}\right) \mid m_{1}\left(S_{1}\right) \geq G_{m_{1}\left(S_{1}\right)}^{-1}(p)\right] & =\mathrm{E}\left[\mathrm{E}\left[X \mid S_{1}\right] \mid S_{1} \geq G^{-1}(p)\right] \\
& =\mathrm{E}\left[X \mid S_{1} \geq G^{-1}(p)\right] .
\end{aligned}
$$

As $S_{1}$ and $S_{2}$ are identically distributed, we see that $m_{1}\left(S_{1}\right) \preceq_{\mathrm{cx}} m_{2}\left(S_{2}\right)$ holds if and only if the inequality

$$
\mathrm{E}\left[X \mid S_{1} \geq t\right] \leq \mathrm{E}\left[X \mid S_{2} \geq t\right]
$$

is valid for all $t$, which is the case if $\left(X, S_{1}\right) \preceq_{(2,1)}\left(X, S_{2}\right)$ by virtue of (3.1).

Note that condition (3.2) also appears in Muliere and Petrone (1992) in their study of dependence orderings based on generalized Lorenz curves.

\section{The $L^{2}$ distance between the signal and conditional expectation}

The stochastic inequality $\left(X, S_{1}\right) \preceq_{(2,1)}\left(X, S_{2}\right)$ means that $S_{2}$ is a better, or more informative, signal for $X$ than $S_{1}$. The next result formalizes this intuitive idea by showing that $m_{2}\left(S_{2}\right)$ is closer to $X$ than $m_{1}\left(S_{1}\right)$ in the $L^{2}$ distance.

Proposition 4.1. If $s \mapsto m_{i}(s)$ is nondecreasing for $i=1,2$ then

$$
\left(X, S_{1}\right) \preceq_{(2,1)}\left(X, S_{2}\right) \Rightarrow \mathrm{E}\left[\left(X-m_{2}\left(S_{2}\right)\right)^{2}\right] \leq \mathrm{E}\left[\left(X-m_{1}\left(S_{1}\right)\right)^{2}\right],
$$

that is, $X$ is closer to $m_{2}\left(S_{2}\right)$ in the $L^{2}$-norm.

Proof. The result is a direct consequence of Proposition 3.1 since

$$
\operatorname{var}[X]=\mathrm{E}\left[\operatorname{var}\left[X \mid S_{i}\right]\right]+\operatorname{var}\left[m_{i}\left(S_{i}\right)\right] \quad \text { and } \quad \mathrm{E}\left[\operatorname{var}\left[X \mid S_{i}\right]\right]=\mathrm{E}\left[\left(X-m_{i}\left(S_{i}\right)\right)^{2}\right]
$$

hold for $i=1,2$, and $m_{1}\left(S_{1}\right) \preceq \mathrm{cx} m_{2}\left(S_{2}\right)$ implies that $\operatorname{var}\left[m_{1}\left(S_{1}\right)\right] \leq \operatorname{var}\left[m_{2}\left(S_{2}\right)\right]$. 
Note that the ' $\preceq_{(2,1)}$ '-ranking needed in this result is rather weak. Most parametric families of bivariate distributions are ' $\preceq_{(1,1)}$ '-monotonic in their parameters so that the result of Proposition 3.1 generally applies.

\section{Acknowledgements}

The author would like to thank Professors Esther Frostig and Christian Genest for interesting discussions on topics related to the contents of the present paper. The author would also like to express his gratitude to an anonymous referee whose comments have been useful in revising a previous version of the present work.

The financial support of the Banque Nationale de Belgique under grant 'Risk Measures and Economic Capital', and of the Onderzoeksfonds K.U. Leuven (GOA/07: Risk Modeling and Valuation of Insurance and Financial Cash Flows, with Applications to Pricing, Provisioning and Solvency) is gratefully acknowledged.

\section{References}

Denuit, M., Lefèvre, Cl. And Mesfioui, M. (1999). A class of bivariate stochastic orderings with applications in actuarial sciences. Insurance Math. Econom. 24, 31-50.

Denuit, M., LefÈvre, CL. AND ShaKed, M. (1998). The $s$-convex orders among real random variables, with applications. Math. Inequal. Appl. 1, 585-613.

Denuit, M., Dhaene, J., Goovaerts, M. J. and KaAs, R. (2005). Actuarial Theory for Dependent Risks: Measures, Orders and Models. John Wiley, New York

GanuZa, J.-J. And Penalva, J. S. (2010). Signal orderings based on dispersion and the supply of private information in auctions. Econometrica 78, 1007-1030.

Mizuno, T. (2006). A relation between positive dependence of signal and variability of conditional expectation given signal. J. Appl. Prob. 43, 1181-1185.

Muliere, P. And Petrone, S. (1992). Generalized Lorenz curve and monotone dependence orderings. Metron 50, 19-38.

Shaked, M. and Shanthikumar, J. G. (2007). Stochastic Orders. Springer, New York. 